\title{
Host Range Limitation of a Pseudorecombinant Virus Produced by Two Distinct Bipartite Geminiviruses
}

\author{
Petra Höfer, Margit Engel, Holger Jeske, and Thomas Frischmuth \\ Biologisches Institut, Lehrstuhl für Molekularbiologie und Virologie der Pflanzen, Universität Stuttgart, \\ Pfaffenwaldring 57, 70550 Stuttgart, Germany \\ Accepted 5 August 1997.
}

\begin{abstract}
The production of pseudorecombinant viruses following exchange of cloned genomic components of the distinct geminiviruses Sida golden mosaic virus (SiGMV-Co) and Abutilon mosaic virus (AbMV) has been investigated. The pseudorecombinant virus produced by DNA A of AbMV and DNA B of SiGMV-Co was infectious in various host plants, although showing a limited host range compared with the two parental viruses. The reciprocal exchange of genomic components did not result in an infectious pseudorecombinant virus. However, in leaf disks SiGMV-Co DNA A was able to trans-replicate the heterogenomic AbMV DNA B component.
\end{abstract}

Additional keywords: pseudorecombination, trans-replication.

Geminiviruses are small plant viruses with circular, singlestranded DNA genomes encapsidated in twinned (geminate) particles (Harrison 1985). Members have been divided into three subgroups (Murphy et al. 1995). The majority of group members that infect dicotyledonous plants have bipartite genomes (DNAs A and B). DNA A encodes the coat protein $(A V 1)$ as well as proteins required for replication $(A C 1)$, gene regulation $(A C 2)$, and replication enhancement (AC3) (for review see Lazarowitz 1992; Timmermans et al. 1994). DNA B is essential for disease production but plays no role in DNA replication. The two gene products ( $B V 1$ and $B C 1)$ encoded by this component are involved in virus spread throughout the plant, symptom production, and host range (Ingham and Lazarowitz 1993; Sanderfoot and Lazarowitz 1995; Timmermans et al. 1994; Noueiry et al. 1994; Ingham et al. 1995; Schaffer et al. 1995).

Although bipartite geminiviruses are closely related, the production of viable pseudorecombinants by reassortment of infectious cloned components is generally limited to strains of a particular virus (Stanley et al. 1985; Lazarowitz 1991; von Arnim and Stanley 1992; Frischmuth et al. 1993; Sung and Coutts 1995b) because of the highly specific nature of the interaction of $A C l$ with the origin of viral replication (Fontes et al. 1994a, 1994b). As an exception, a viable pseudorecombination between the two distinct geminiviruses tomato mottle

Corresponding author: Thomas Frischmuth

Telephone: +49-711-6855075; Fax: +49-711-6855096

E-mail: thomas.frischmuth@po.uni-stuttgart.de virus (ToMoV; Abouzid et al. 1992) and bean dwarf mosaic virus (BDMV; Hidayat et al. 1993) has been reported (Gilbertson et al. 1993).

A second obstacle for the production of viable pseudorecombinants might be the inability of DNA B gene products to mediate the movement of heterogenomic DNA A components. For a number of bipartite geminiviruses complementation of DNA B gene products has been demonstrated following coinoculation with both genomic components of one virus and DNA A of another virus (Frischmuth et al. 1993; Sung and Coutts 1995b). This ability to mediate the movement of a heterogenomic DNA A component seems to be dependent on the origin of the virus, in general New or Old World (Frischmuth et al. 1993). Here, we describe a pseudorecombination between SiGMV-Co and AbMV, two distinct geminiviruses from the New World.

The cloning of infectious genomic components A and B of SiGMV-Co (Höfer et al. 1997) and AbMV (Frischmuth et al. 1990, 1993) has been described. For infection experiments genomic components were introduced into various hosts by stem agroinoculation as described in Stanley et al. (1990) or by rub inoculation of $10 \mu \mathrm{l}$ of Agrobacterium tumefaciens suspension, containing cloned genomic components, onto Carborundum-dusted Nicotiana benthamiana D. leaves (leaf agroinoculation). Infection of plants was evaluated by symptom appearance, Southern blot analysis, and polymerase chain reaction (PCR). Southern blotting was performed with a probe specific for the DNA A or B component of SiGMV-Co and AbMV. Because of the close relationship the probes recognize both viruses. PCR was performed with specific primers for SiGMV-Co (SiGMV19: 5'-TCA AAC TTG CTG ATT CTA T$3^{\prime}$ and SiGMV20: 5'-GAG GGC TCA ACT GGA GTA-3') and AbMV (AbMV9: 5'-TTG GAG GTC TTT ATA TAC-3' and AbMV10: 5'-TAC TCC AAT TAC ATG AGC-3'). The primers are located within the common region sequences of DNA A and B in opposite orientation and, therefore, are capable of amplifying both genomic components of SiGMV-Co or AbMV. The ability of DNA A components to trans-replicate heterogenomic DNA B was assayed in $N$. benthamiana leaf disks as described in Frischmuth and Stanley (1991) and analyzed by PCR and Southern blotting (Fig. 1B and C).

SiGMV-Co and AbMV were stem agroinoculated in Nicotiana tabacum L. 'Xanthi nc', Malva parviflora L., Sida rhombifolia L., N. benthamiana, Phaseolus vulgaris L., and 
A

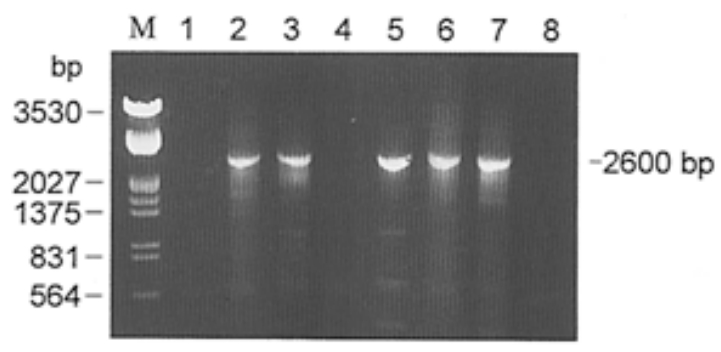

B

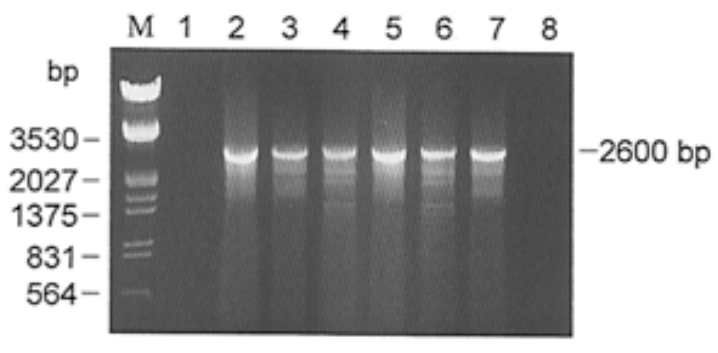

C

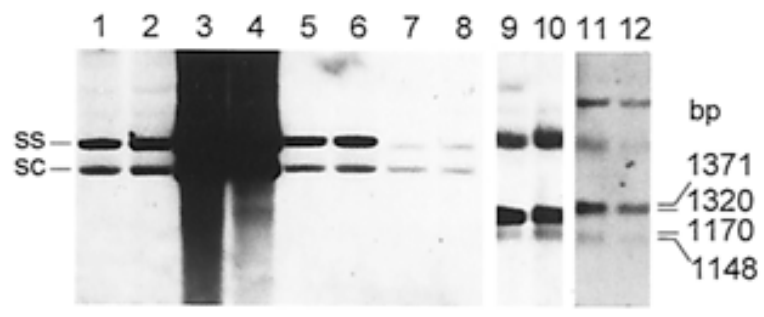

Fig. 1. Polymerase chain reaction (PCR) analysis of stem agroinoculated Nicotiana benthamiana $(\mathbf{A})$ and leaf disks of $N$. benthamiana $(\mathbf{B})$ as well as Southern blot analysis of leaf disks of $N$. benthamiana $(\mathbf{C})$. PCR was performed in both cases with primers specific for Sida golden mosaic virus (SiGMV-Co; lanes 1, 3, 5, and 7) and with primers specific for Abutilon mosaic virus (AbMV; lanes 2, 4, 6, and 8). Southern blot analysis was performed with a Sst I (1045)-SstI (2552) fragment of SiGMV-Co DNA B as a probe. A, Nucleic acids were extracted from newly expanding upper leaves of AbMV DNA A and B (lanes 1 and 2), SiGMV-Co DNA A and B (lanes 7 and 8), SiGMV-Co DNA A and AbMV DNA B (lanes 3 and 4), and AbMV DNA A and SiGMV-Co DNA B (lanes 5 and 6) stem-agroinoculated plants. B, Nucleic acids were extracted from leaf disks in the same order described in A. The approximate size of viral genomic DNA and selected sizes of a HindIII/EcoRI digested $\lambda$ DNA molecular weight marker (lane M) are indicated. C, Nucleic acids were extracted from 2 individual leaf disks inoculated with AbMV DNA A and SiGMV-Co DNA B (lanes 1 and 2), SiGMV-Co DNA A and B (lanes 3 and 4), AbMV DNA A and B (lanes 5 and 6), and SiGMV-Co DNA A and AbMV DNA B (lanes 7 and 8). Nucleic acids extracted from AbMV DNA A and SiGMV-Co DNA B (lanes 9 and 10) and SiGMV-Co DNA A and AbMV DNA B (lanes 11 and 12) inoculated leaf disks were digested with SalI. Samples of nucleic acids were run on $1 \%$ agarose gels in $0.5 \times$ Tris-borate-EDTA resulting in single-stranded DNA migration slower than supercoiled DNA (Stanley et al. 1990). Five micrograms of total nucleic acids was analyzed in lanes 1 to $10,10 \mu \mathrm{g}$ in lanes 11 and 12 . Viral single-stranded (SS) and supercoiled (SC) DNA forms are indicated. The expected sizes of SiGMV-Co DNA B and AbMV DNA B after digestion with SalI are indicated.
Datura stramonium L. (Table 1). The general infection symptoms of SiGMV-Co are described in Höfer et al. (1997). $N$. benthamiana, $N$. tabacum cv. Xanthi nc, and P. vulgaris infected with AbMV exhibited weak leaf curling and stunting whereas the infection symptoms in $D$. stramonium, $M$. parviflora, and S. rhombifolia were as severe as those of SiGMVCo.

These common hosts of SiGMV-Co and AbMV were stem agroinoculated with pseudorecombinant viruses produced by mixing of genomic components A and B. None of the SiGMVCo DNA A and AbMV DNA B inoculated plants exhibited symptoms (Table 1). The pseudorecombinant virus consisting of AbMV DNA A and SiGMV-Co DNA B produced infection symptoms in $N$. tabacum cv. Xanthi nc, $N$. benthamiana, and $P$. vulgaris but not in $M$. parviflora, S. rhombifolia, and $D$. stramonium (Table 1). The symptoms of the pseudorecombinant virus were of an intermediate type between AbMV and SiGMV-Co, mild stunting and leaf curling. Symptoms of an intermediate type were also observed after inoculation of plants with a pseudorecombinant virus produced by ToMoV and BDMV (Gilbertson et al. 1993). Newly developing leaves of plants with and without symptoms were analyzed by Southern blot (data not shown) and PCR (Fig. 1A). In AbMV DNA A and SiGMV-Co DNA B inoculated $N$. benthamiana both genomic components were detected (Fig. 1A, lanes 5 and 6). The pseudorecombination between SiGMV-Co DNA A and AbMV DNA B showed no symptoms in N. benthamiana (Table 1). However, systemic spread of SiGMV-Co DNA A was detected in these inoculated plants by Southern blotting (data not shown) and PCR (Fig. 1A, lane 3). Symptomless systemic spread of DNA A component following agroinoculation has been observed for several bipartite geminiviruses (Klinkenberg and Stanley 1990; Evans and Jeske 1993; Buragohain et al. 1994). Systemic spread of the DNA A component of SiGMV-Co in the absence of a DNA B component was also observed following stem agroinoculation of $N$. benthamiana (Fig. 2A, lane 3). In contrast, following leaf agroinoculation of $N$. benthamiana, replication of SiGMV-Co DNA A was detected in the inoculated leaf by Southern blot (Fig. 2A, lane 1) but no systemic spread of SiGMV-Co DNA A was observed (Fig. 2A, lane 2). Leaf agroinoculation of SiGMV-Co DNA A and B (Fig. 2A and B, lanes 4 and 5), AbMV DNA A and B, and AbMV DNA A and SiGMV-Co DNA B (data not shown) resulted in systemic infection. Following leaf agroinoculation of a mixture of SiGMV-Co DNA A and AbMV DNA B no systemic spread of SiGMV-Co DNA A or AbMV DNA B was observed by PCR and Southern blot (data not shown). Therefore, we conclude that the systemic spread of SiGMV-Co DNA A after stem agroinoculation with AbMV DNA B is due to the inoculation technique. Similar results have been reported for potato yellow mosaic virus (PYMV; Coutts et al. 1991). Systemic spread of PYMV DNA A was observed following stem agroinoculation but not after biolistic delivery of DNA (Sung and Coutts 1995a).

The inability of SiGMV-Co DNA A and AbMV DNA B to produce a viable pseudorecombinant virus might be due to the inability of SiGMV-Co DNA A to trans-replicate heterogenomic AbMV DNA B. In $N$. benthamiana leaf disks, SiGMV-Co DNA A was able to trans-replicate the heterogenomic AbMV DNA B (Fig. 1B, lanes 3 and 4; Fig. 1C, 
lanes 7, 8, 11, and 12), although at a lower level than AbMV and SiGMV-Co trans-replicate their own DNA B component (compare Figure 1C lanes 7 and 8 with lanes 3, 4, 5, and 6). Trans-replication of SiGMV-Co DNA A and AbMV DNA B was also observed in the agroinoculated leaves of $N$. benthamiana (data not shown).

Because SiGMV-Co DNA A was able to trans-replicate AbMV DNA B, other viral gene products involved in spread, gene regulation, or virus structure might be unable to interact with each other or the heterogenomic DNA. Systemic spread of heterogenomic DNA components has been observed in coinoculation experiments for bipartite geminiviruses that were not able to produce viable pseudorecombinant viruses (Frischmuth et al. 1993; Sung and Coutts 1995b), implying that viral spread of heterogenomic components can be supported by distinct viruses.

One of the requirements for trans-replication is interaction of $A C 1$ with the origin of viral replication within the common region (Fontes et al. 1994a, 1994b). Homology analysis of the common region showed a close relationship between AbMV and SiGMV-Co (Höfer et al. 1997). A similar clustering was observed between BDMV and ToMoV (Abouzid et al. 1992), the only other two distinct geminviruses for which pseudorecombination has been demonstrated (Gilbertson et al. 1993). The pseudorecombinant virus consisting of BDMV DNA A and ToMoV DNA B lost its viability after three serial passages in $N$. benthamiana whereas the reciprocal exchange of genomic components resulted in a highly pathogenic pseudorecombinant virus due to inter-molecular recombination between the common regions (Hou and Gilbertson 1996). Here, we showed that AbMV DNA A and SiGMV-Co DNA B produce a viable pseudorecombinant virus whereas the reciprocal exchange of genomic components was not infectious in plants, although trans-replication was observed. As suggested by Frischmuth and Stanley (1991), suitably high levels of DNA A and B are necessary during early phases of infection to ensure that subsequent systemic infection is initiated by both genomic components. Therefore, the low accumulation of AbMV DNA B trans-replicated by SiGMV-Co DNA A (Fig. 1C, lanes 7 and 8) might be the primary cause for nonsystemic infection of this pseudorecombinant virus.

The pseudorecombinant virus formed by DNA A of AbMV and DNA B of SiGMV-Co was viable in a limited range of hosts, compared with those of both parental viruses (Table 1), indicating that host-encoded factors are necessary for the production of a viable pseudorecombinant virus. Stenger et al. (1994) observed that recombinant beet curly top virus showed different pathogenicity and virulence in $N$. benthamiana and Beta vulgaris L., also indicating influences of host-encoded
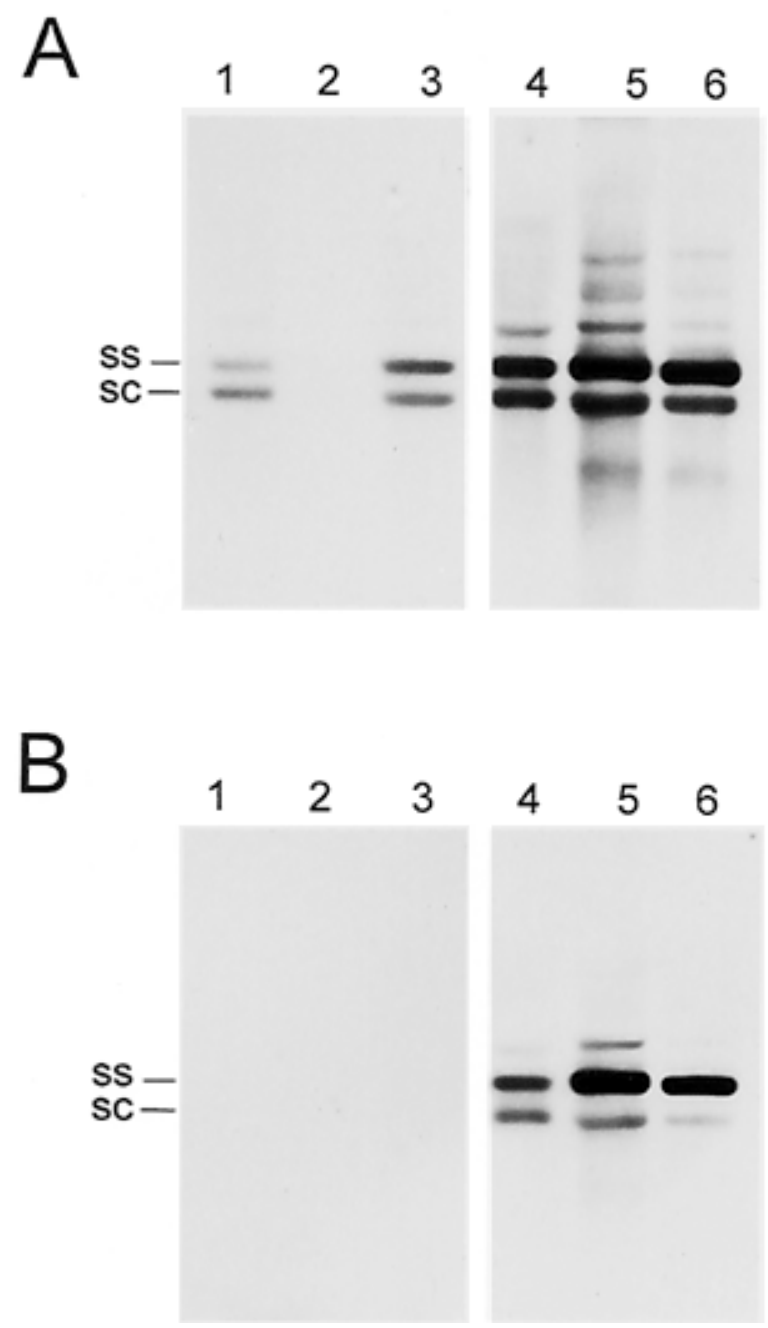

Fig. 2. Southern blot analysis of leaf-agroinoculated Nicotiana benthamiana plants. The membranes were hybridized with probes specific for Sida golden mosaic virus DNA A [SiGMV-Co DNA A EcoRV (564)-PstI (2479) fragment, A] and DNA B [SiGMV-Co DNA B SstI(1045)-SstI (2552) fragment, B]. Nucleic acids were extracted from a SiGMV-Co DNA A agroinoculated leaf (A and $\mathbf{B}$, lane 1), from a newly expanding upper leaf of the same plant ( $\mathbf{A}$ and $\mathbf{B}$, lane 2) as well as from a systemic leaf of a stem-agroinoculated plant (A and $\mathbf{B}$, lane 3). Nucleic acids were extracted from a SiGMV-Co DNA A and B agroinoculated leaf (A and $\mathbf{B}$, lane 4), from a newly expanding upper leaf of the same plant (A and B, lane 5) and from a systemic leaf of a SiGMV-Co DNA A and B stem agroinoculated plant (A and $\mathbf{B}$, lane 6). Viral single-stranded (SS) and supercoiled (SC) DNA forms are indicated.

Table 1. Stem agroinoculation of various host plants with cloned genomic components

\begin{tabular}{|c|c|c|c|c|c|c|}
\hline \multirow[b]{2}{*}{ Virus } & \multicolumn{6}{|c|}{ Host species } \\
\hline & $\begin{array}{l}\text { Nicotiana taba- } \\
\text { cum cv. Xanthi }\end{array}$ & Malva parviflora & Sida rhombifolia & N. benthamiana & $\begin{array}{c}\text { Phaseolus } \\
\text { vulgaris }\end{array}$ & $\begin{array}{c}\text { Datura } \\
\text { stramonium }\end{array}$ \\
\hline SiGMV-Co A+B & $6 / 6^{\mathrm{a}}$ & $6 / 6$ & $3 / 20$ & $6 / 6$ & $6 / 6$ & $4 / 5$ \\
\hline $\mathrm{AbMV} A+\mathrm{B}$ & $5 / 6$ & $6 / 6$ & $2 / 10$ & $5 / 6$ & $4 / 6$ & $3 / 5$ \\
\hline SiGMV-Co A + AbMV B & $0 / 40$ & $0 / 18$ & $0 / 40$ & $0 / 40$ & $0 / 12$ & $0 / 5$ \\
\hline AbMV A + SiGMV-Co B & $4 / 6$ & $0 / 18$ & $0 / 40$ & $5 / 6$ & $3 / 6$ & $0 / 5$ \\
\hline
\end{tabular}

\footnotetext{
${ }^{a}$ Infectivity (infected/inoculated) was assayed in all hosts by visible symptoms, Southern blot, and polymerase chain reaction analysis. Infectivity num-
} bers are from one representative experiment out of six. 
factors. Taken together, pseudorecombination between bipartite geminiviruses is a complex phenomenon that involves interactions among virus- and host-encoded factors as well as viral DNA components.

\section{ACKNOWLEDGMENTS}

Virus clones were held according the Gentechnik Gesetz (license nos. 76-14/8829.02/Uni.S.01.01-5 and 76-14/8829.02/Uni.S.01.04-2). The work was supported by a DFG grant to T. F. (Fr. 1122/1-1).

\section{LITERATURE CITED}

Abouzid, A. M., Polston, J. E., and Hiebert, E. 1992. The nucleotide sequence of tomato mottle virus, a new geminivirus isolated from tomatoes in Florida. J. Gen. Virol. 73:3225-3229.

Buragohain, A. K., Sung, Y. K., Coffin, R. S., and Coutts, R. H. A. 1994. The infectivity of dimeric potato yellow mosaic geminivirus clones in different hosts. J. Gen. Virol. 75:2857-2861.

Coutts, R. H. A., Coffin, R. S., Roberts, E. J. F., and Hamilton, W. D. O. 1991. The nucleotide sequence of the infectious cloned DNA components of potato yellow mosaic virus. J. Gen. Virol. 72:1515-1520.

Evans, D., and Jeske, H. 1993. DNA B facilitates, but is not essential for, the spread of Abutilon mosaic virus in agroinoculated Nicotiana benthamiana. Virology 194:752-757.

Fontes, E. P. B., Eagle, P., Sipe, P., Luckow, V. A., and Hanley-Bowdoin, L. 1994a. Interaction between a geminivirus replication protein and origin DNA is essential for viral replication. J. Biol. Chem. 269:84598465.

Fontes, E. P. B., Gladfelter, H. J., Schaffer, R. L., Petty, I. T. D., and Hanley-Bowdoin, L. 1994b. Geminivirus replication origins have a modular organization. Plant Cell 6:405-416.

Frischmuth, T., Roberts, S., von Arnim, A., and Stanley, J. 1993. Specificity of bipartite geminivirus movement proteins. Virology 196:666673.

Frischmuth, T., and Stanley, J. 1991. African cassava mosaic virus DI DNA interferes with the replication of both genomic components. Virology 183:539-544.

Frischmuth, T., Zimmat, G., and Jeske, H. 1990. The nucleotide sequence of Abutilon mosaic virus reveals prokaryotic as well as eukaryotic features. Virology 178:461-468.

Gilbertson, R. L., Hidayat, S. H., Paplomatas, E. J., Rojas, M. R., Hou, Y.-M., and Maxwell, D. P. 1993. Pseudorecombination between infectious cloned DNA components of tomato mottle and bean dwarf mosaic geminiviruses. J. Gen. Virol. 74:23-31.

Harrison, B. D. 1985. Advances in geminivirus research. Annu. Rev. Phytopathol. 23:55-82.

Hidayat, S. H., Gilbertson, R. L., Hanson, S. F., Morales, F. J., Ahlquist, P., Russell, D. R., and Maxwell D. P. 1993. Complete nucleotide sequences of the infectious cloned DNAs of bean dwarf mosaic geminivirus. Phytopathology 83:181-187.

Höfer, P., Engel, M., Jeske, H., and Frischmuth, T. 1997. Nucleotide sequence of a new bipartite geminivirus isolated from the common weed Sida rhombifolia in Costa Rica. J. Gen. Virol. 78:1785-1790.

Hou, Y.-M., and Gilbertson R. L. 1996. Increased pathogenicity in a pseudorecombinant bipartite geminivirus correlates with intermolecular recombination. J. Virol. 70:5430-5436.

Ingham, D. J., and Lazarowitz, S. G. 1993. A single missense mutation in the BR1 movement protein alters the host range of the squash leaf curl geminivirus. Virology 196:694-702.

Ingham, D. J., Pascal, E., and Lazarowitz, S. G. 1995. Both bipartite geminivirus movement proteins define viral host range, but only BL1 determines viral pathogenicity. Virology 20:7191-204.

Klinkenberg, F. A., and Stanley, J. 1990. Encapsidation and spread of African cassava mosaic virus DNA A in the absence of DNA B when agroinoculated to Nicotiana benthamiana. J. Gen. Virol. 71:14091412.

Lazarowitz, S. G. 1991. Molecular characterization of two bipartite geminiviruses causing squash leaf curl disease: Role of viral replication and movement functions in determining host range. Virology 180:70-80.

Lazarowitz, S. G. 1992. Geminiviruses: Genome structure and gene function. Crit. Rev. Plant Sci. 11:327-349.

Murphy, F. A., Fauquet, C. M., Bishop, D. H. L., Ghabrial, S. A., Jarvis, A. W., Martelli, G. P., Mayo, M. A., and Summers, M. D. 1995. Virus taxonomy. Arch. Virol. Suppl. 10:158-165.

Noueiry, A. O., Lucas, W. J., and Gilbertson, R. L. 1994. Two proteins of a plant DNA virus coordinate nuclear and plasmodesmal transport. Cell 76:925-932.

Sanderfoot, A. A., and Lazarowitz, S. G. 1995. Cooperation in viral movement: The geminivirus BL1 movement protein interacts with BR1 and redirects it from the nucleus to the cell periphery. Plant Cell 7:1185-1194.

Schaffer, R. L., Miller, C. G., and Petty, I. T. D. 1995. Virus and hostspecific adaptations in the BL1 and BR1 genes of bipartite geminiviruses. Virology 214:330-338.

Stanley, J., Frischmuth, T., and Ellwood, S. 1990. Defective viral DNA ameliorates symptoms of geminiviruses infection in transgenic plants. Proc. Natl. Acad. Sci. USA 87:6291-6295.

Stanley, J., Townsend, R., and Curson, S. J. 1985. Pseudorecombinants between cloned DNAs of two isolates of cassava latent virus. J. Gen. Virol. 66:1055-1061.

Stenger, D. C., Davis, K. R., and Bisaro, D. M. 1994. Recombinant beet curly top virus genomes exhibit both parental and novel phenotypes. Virology 200:677-685.

Sung, Y. K., and Coutts, R. H. A. 1995a. Mutational analysis of potato yellow mosaic geminivirus. J. Gen. Virol. 76:1773-1780.

Sung, Y. K., and Coutts, R. H. A. 1995b. Pseudorecombination and complementation between potato yellow mosaic geminivirus and tomato golden mosaic geminivirus. J. Gen. Virol. 76:2809-2815.

Timmermans, M. C. P., Das, O. P., and Messing, J. 1994. Geminiviruses and their uses as extrachromosomal replicons. Annu. Rev. Plant Physiol. Plant Mol. Biol. 45:79-112.

von Arnim, A., and Stanley, J. 1992. Determinants of tomato golden mosaic virus symptom development located on DNA B. Virology 186: 286-293. 